hepatitis, neuropsychiatric disorders and Stevens-Johnson syndrome, ${ }^{3}$ and so the risk-benefit balance in uncomplicated cases in the community favours not giving it.

\section{FERGUS DIGNAN}

Civilian medical practitioner, MOD Boscombe Down, Salisbury

\section{References}

1 Department of Health. Pandemic H1N1 2009 influenza: clinical management guidelines for adults and children, 2009. London: DOH, 2009.

2 Health Protection Agency. HPA guidance on use of antiviral agents for the treatment and prophylaxis of influenza, 2011-12 (version 3). London: HPA, 2011.

3 British National Formulary 62. London: BNF,2011;5.3.5:408.

\section{Clinical and scientific letters}

Letters not directly related to articles published in Clinical Medicine and presenting unpublished original data should be submitted for publication in this section. Clinical and scientific letters should not exceed 500 words and may include one table and up to five references.

The current system for prescribing antiretroviral therapy puts HIVinfected patients at risk of serious drug-drug interactions: is now the time for a paradigm shift in HIV care delivery?

\section{Introduction}

The UK Health Protection Agency estimates that by 2012 there will be over 100,000 persons living with $\mathrm{HIV},{ }^{1}$ with one in five being over 50 years of age. ${ }^{2}$ At present approximately $80 \%$ of patients receiving care in the UK are prescribed antiretroviral medication, which is currently dispensed from HIV services. ${ }^{2}$ All other medication is ordinarily prescribed by the patients' GP. Co-morbidities are increasingly recognised among this ageing HIV cohort and approximately one in six medications prescribed in primary care for HIV-positive individuals has the potential for major drug-drug interactions with antiretroviral medication, primarily mediated via cytochrome $\mathrm{P} 450$ metabolism. ${ }^{3}$ For example, simvastatin coadministered with ritonavir can result in markedly increased concentrations of simvastatin, predisposing patients to myopathies and rhabdomyolysis., ${ }^{4,5}$ It is essential that there is good communication between all prescribers in order to prevent avoidable morbidity and mortality.

We report two HIV-positive individuals receiving antiretroviral therapy who were inadvertently prescribed by their primary care physician additional medication known to have significant life-threatening drug-drug interactions.

\section{Case 1}

A 34-year-old man complained of chronic cough. Following an outpatient ear, nose and throat review, a letter was sent to his GP recommending a fluticasone nasal spray. No copy of the letter was sent to the HIV physician. The patient had been HIVpositive for 10 years and was receiving antiretroviral therapy (lopinavir/ritonavir); CD4 count was 670 (normal $>270 \times 10^{6}$ cells/l) and plasma HIV viral load was undetectable. At routine outpatient review by the HIV physician, the patient disclosed that he had started using a fluticasone nasal spray eight days previously. He was advised to stop it immediately due to the risk of developing Cushing's syndrome. The HIV physician contacted the GP, alerting them to the interaction between fluticasone and protease inhibitors. A beclomethasone nasal spray was subsequently prescribed.

\section{Case 2}

A 42-year-old man, who was known to have HIV infection for five years, was admitted to hospital with sudden onset visual disturbance, dysarthria and headache. Cranial magnetic resonance imaging demonstrated an acute infarction of the right middle cerebral artery. At this time, the CD4 count was $670 \times 10^{6}$ cells/l with an undetectable plasma viral load. His HIV medication included fosamprenavir/ritonavir, tenofovir and emtricitabine. Pravastatin was also prescribed by the HIV clinic for antiretroviral-therapy-induced hypercholesterolaemia. During hospitalisation, he was found to have diabetes mellitus and commenced gliclazide in addition to aspirin and clopidogrel. A discharge summary was sent to the GP but not the HIV clinic. At routine outpatient review six months later, the HIV physician discovered that the patient's GP had changed lipidlowering medication from pravastatin to simvastatin following discharge. The patient had no clinical or biochemical evidence of myopathy or rhabdomyolysis. The HIV physician contacted the GP alerting them to the interaction between simvastatin and protease inhibitors. Atorvastatin was subsequently prescribed. 


\section{Discussion}

These cases highlight that HIV patients are being put at risk of serious drug errors due to prescribing of medication with important drug-drug interactions by different physicians in different locations. It is common practice for HIV clinics to write to GPs with details of prescribed medication but is not standard practice for GPs or other services to routinely write to HIV services. Our standard letter also contains a 'footer' highlighting that drug-drug interactions are common. In view of the concern of prescribing errors, we introduced a policy of requesting a faxed summary of medication from primary care within the standard letter and subsequently reviewed the impact of this policy. Between 3 September and 15 November 2010, we requested prescribing data from GPs for 99 consecutive HIVinfected patients attending a large HIV outpatient clinic in North Central London. An amalgamated list of medications prescribed for each patient by primary care and the HIV service was examined for potential drug-drug interactions. Fifty-six GPs provided prescribing data. The medication lists from GPs and the HIV service were identical in three cases. No patients had life-threatening drug-drug interactions. However, 33 had potentially significant drug-drug interactions and, for almost all, the HIV service was not aware the patient was being prescribed the implicated drug.

Our findings demonstrate that there is a lack of documented awareness of drugs prescribed in each service, which is responsible for regular dispensing of medication, despite efforts to improve communication. It is essential that the patients and all clinicians involved in their care are aware of the potential for drug-drug interactions and ensure that up-to-date prescribing information is shared. However, even if this happens, our highlighted cases demonstrate that inadvertent changes to prescribing may occur in between visits to the HIV service by physicians who may be unfamiliar with the complex drug interactions of HIV therapy. The Royal Pharmaceutical Society has recently published guidance due to concerns of poor communication of prescribing information between healthcare providers. ${ }^{6}$ This raises the question as to whether all medication (including HIV drugs) should be prescribed via primary care, thus providing a single site for all prescribing. While this would reduce inadvertent prescribing errors due to communication problems, this model of care is, to date, untested in the UK.

Since the start of the HIV epidemic patients have been cared for in specialist services. However, due to the success of antiretroviral therapy, HIV is now considered a long term condition, of which the majority are conditions in which care is delivered in the community. The British HIV Association have recently developed a position statement on greater engagement with primary care that highlights many of the complex issues that would need addressing to facilitate a change in the model of care. ${ }^{7}$

However, while HIV services continue to prescribe HIV medication, greater awareness is needed of prescribing between specialist services and primary care. We recommend that details of drugs prescribed elsewhere are recorded and updated on the primary care prescribing database to avoid untoward prescribing errors.

GG WHITLOCK A PATEL

SG EDWARDS PD BENN

Department of Genitourinary Medicine, Camden Provider Services PCT, The Mortimer Market Centre, London, UK

RF MILLER

Centre for Sexual Health and HIV

Research, University College London

Medical School, University College London,

London, UK

\section{References}

1 Health Protection Agency. 30 years on: people living with HIV in the UK about to reach 100,000. www.hpa.org.uk/hpr/ archives/2011/news2211.htm [Accessed 31 July 2011].

2 Health Protection Agency. Numbers accessing care: national overview. www.hpa. org.uk/web/Aweb\&HPAwebStandard/ PAweb_C/1203064766492 [Accessed 31 July 2011].
3 Evans HE, Tsourapas A, Mercer CH et al. Primary care consultation and costs among HIV-positive individuals in UK primary care 1995-2005: a cohort study. Sex Transm Inf 2009; 85:543-9.

4 Patel BL, Choudhury M. Rhabdomyolysis with simvastatin. BMJ Case Reports 2011; doi:10.1136/bcr.12.2009.2552.

5 Foisy MM, Yakiwchuk EM, Chin I, Singh AE. Adrenal suppression and Cushing's syndrome secondary to an interaction between ritonavir and fluticasone: a review of the literature. HIV Medicine 2008;9:389-96.

6 Royal Pharmaceutical Society. Keeping patients safe when they transfer between care providers - getting the medicines right. London: RPS, 2011. www.rpharms.com/ medicines-safety/getting-the-medicinesright.asp [Accessed 31 July 2011].

7 British HIV Association. The future role of primary and community care in HIV. www. bhiva.org/documents/Publications/ PositionStatement.pdf [Accessed 31 July 2011].

\section{The death of diagnosis}

We have observed that junior doctors appear reluctant to attempt a diagnosis nowadays and tend to leave the diagnosis box in the clerking form blank, or record a descriptive term, eg 'collapse ?cause'. This absence could lead to a delay in appropriate treatment or non-specific use of broadspectrum antibiotics, resulting in iatrogenic complications and prolonged admission. ${ }^{1,2}$ A specific diagnosis would also avoid unnecessary investigations.

This service evaluation examined how frequently acute medical patients receive a diagnosis, as opposed to a clinical epithet, on admission or later during their hospital stay.

Over three days, the records of 100 consecutive patients from the Medical Admissions Unit (MAU) and four general medical wards at a UK provincial teaching hospital were analysed to see if, and at which point during their stay, a definite, provisional or differential diagnosis was recorded. Patient files were reviewed at three time points:

1 after junior review, within four hours of admission

2 after senior review, within 24 hours of admission

3 at the time of discharge from hospital, and on the discharge summary. 